\section{THE CROONIAN LECTURES:}

\section{A CONTRIBUTION TO THE STUDY OF THE BLOOD AND THE CIRCULATION.}

\section{Delivered before the Royal College of Physicians of London. \\ By GEORGE OLIVER, M.D., F.R.C.P.}

\section{LECTURE II.}

ANIMAL EXTRACTS.

Some preliminary observations with the arteriometer having afforded me evidence that certain of the animal extracts (such as those of the suprarenal bodies, the thyroid gland, and the brain tissue), when administered to healthy subjects by the mouth modify the calibre of the arteries, I was led to avail myself of the resources of the laboratory in order to determine the matter. In this investigation $I$ had the invaluable co-operation of Professor Schäfer. ${ }^{1}$ The extracts employed were those derived from the suprarenal bodies, the thyroid gland, the spleen, and the pituitary body. They were prepared for the most part with water (in the earlier part of the inquiry with glycerine) and either from the fresh glands, or from glands dried rapidly at $38^{\circ} \mathrm{C}$. The suprarenal extract is the only one of the series which has been investigated in any detail; but as the effects on the blood pressure have been determined in a preliminary way in the others, I will refer to them before describing more particularly the physiological action of the suprarenal extract.

Intravenous injection of thyroid extract produced a marked fall in the blood pressure, although the beats of the heart remained the same in frequency and strength. Therefore it may be inferred that the lowering of the blood pressure was the direct result of increase in the calibre of the arteries--an inference which derives support from the observation which had previously been made with the arteriometer. This direct action on the vascular system may be connected with important functions, such, for instance, as the heat regulating function. The subjects of myxœdema and animals on which thyroidectomy has been performed are particularly prone to suffer from subnormal temperatures. Lorrain Smith has demonstrated that thyroidectomised animals react to change of temperature with abnormal rapidity, as indicated by the shortening of the time required for the augmented emission of carbonic acid which follows exposure to a low temperature. ${ }^{2}$ It is, therefore, not unlikely that in myxœdema the withdrawal of the internal secretion of the thyroid, which acts directly in dilating the vascular system, may in a measure account for the reduction of the temperature so frequently observed and for the development of other signs of lowered vitality. We are not, however, prepared to assert that this effect of thyroid extract on the arterial wall is specific; for we have found that a similar result is obtained by the intravenous injection of decoctions of some other glandular organs, for example, the parotid and submaxillary glands of the dog.

The intravenous injection of spleen extract produces a somewhat characteristic curve of blood pressure, consisting of a preliminary fall succeeded by a gradual rise and by as gradual a return to the normal: The significance of this curve has not been as yet investigated. On the other hand, the pituitary and suprarenal extracts were found to possess the property of exciting powerful contraction of the arteries, and of raising the blood pressure to a very marked degree. The action of both these extracts on the vascular system is not only different in character from that of the thyroid and spleen extracts, but is more energetic. Moreover, though they possess the common property of constricting the arteries, they themselves present certain points which differentiate the one from the other, such as the following:

x. The pituitary extract, though the effect of it is well marked, is less active than the suprarenal; for a much larger dose of it is required to produce the same rise of blood pressure.

2. The pituitary curve of blood pressure rises more slowly, and is maintained for a longer time than the suprarenal curve; the active principle
is, therefore, less readily eliminated from the blood than that of the suprarenal.

7 The 1 esults of this research have appeared in the Journal of Physiology, 2 On some Effects of Thyroidectomy in Animals. by J. Lorrain Smith, M.D.
and Journal of Physiology, vol, xvii, p. 378.
3. The pituitary extract also differs from the supiarenal when injected into a dog with intact vagi in failing to inhibit the heart's action. 4. The pituitary does not possess the characteristic action of suprarenal extract in accelerating the heart's action in an animal with cut vagi, or when atropinised; on the other hand, it then generally diminishes slightly the frequency, and at the same time augments the beats of the The and ventricle.

The action of the two extracts coincides, liowever, in the following respects :

r. The rise of blood pressure is the combined effect of $(a)$ contraction of the arterioles and $(b)$ augmentation of the heart's beats

2. The effect on the heart and the arteries is direct or peripheral, forit occurs equally well with the cord cut or the bulb destroyed, and when system of the frog with the nervous centres destroyed, the flow of fluid is greatly diminished.

3. Boiling for a short time does not destroy the activity of the ex-

The action of suprarenal extract on the circulatory system may be divided into that which affects the heart and that which affects the arteries.

(a) The effects on tine heart have been observed on the isolated frog ventricle and on the mammalian heart, the former being brought under the influence of the extract by perfusion in Ringer's circulating fluid, and the latter by intravenous injection.

The heart of the common frog was tied in the usual manner to a perfusion cannula, and fed through this with the circulating fluid in the apparatus elsewhere described. ${ }^{3}$ It was observed that when the ventricle failed to beat the suprarenal eirculating fluid invariably produced spontaneous contraction; that even when the contractions only recurred after long intervals it accelerated and regulated the rhythm; that when successive groups of contractions (Luciani's groups) appeared with varying asystolic intervals it increased the number of the contractions in each group until the rhythm became uninterrupted; and that when the ventricle was beating feebly it tended to increase the strength of the contractions. Furthermore, it was found that with a certain proportion.of the extract the time of the pause between two successive contractions. is markedly reduced, and with a further proportion of it the pause may. le entirely obliterated, and the beats succeed one another without any apparent interval. A further stage in this action, which obtains with solutions of still greater strength (I per cent.. and upwards), is that the beats begin to run the one into the other, one contraction succeedii $g$ another before the completion of the preceding diastole. The result \& that the dilatation is imperfect, and may ultimately be almost entirely ( $r$ quite abolished, so that eventually the heart stops in systole.

If now, however, the suprarenal circulating fluid is washed away with the ordinary circulating fluid the heart may completely recover, and the same experiment may be repeated several times in the same fashion. The effects of the extract on the mammalian heart were observed in dogs, cats, and rabbits. The contraction of the auricle and ventricle were separately recorded by means of the apparatus described in the paper which has appeared in the Journal of Physiology, vol. xivii, 1895 . The effects produced were found to be entirely different according as. the vagi are intact or are cut or paralysed by atropine. When the vagi are uncut the most striking effect is to slow or even entirely to abolish for a time the contraction of the auricles. Their action, after the period of latency necessary for the passage of the solution to the heart. is rapidly stopped in diastole; in other words, they are powerfully.jnhibited: But usuadly this inhibition of the auricles is preceded by a short period of augmentation. When the auricles are inhibited, the ventricles continue to beat with an independent, slow rhythm. On the other hand, when the vagi are cut or when the animal is atropinised the effect; of the extract: an'the heart is exactly reversed-namely, the contractions of the auricle.and enticle are markedis augment of the extract is not derived from direct action upon the heart.

It would seem, therefore, from these observations : that suprarenal extract exerts a powerfully stimulant action on on the heart.

(b) The Effects on the Arteries.-That contraction of the arteries and arterioles is a prominent effect of the extract has been abundantly demonstrated by the application of Mosso's plethysmograph to the limbs and of Roy's onoometer to the spleen and kidney; by perfusing Ringer's circulating fluid containing the extract through the arterial system of the frog after destruction of the nervous system, and noting the result of the passage of the extract in altering the rate of flow through the blood vessels, and by direct ocular evidence of the large and small blood vessels. This contraction is so great as to produce an enormous diminution in the volume of an organ such as a limb, or the spleen, or the kidney, and in the case of the frog with its nervous system destroyed, almost complete cessation of the flow of circulating fluid through the arterioles. Consequently the blood pressure rises very considerably, even though concomitant vagus action causes a great diminution in the rate of the heart's action. Though this increase of the blood pressure is large while the vagi remain uncut it becomes enormous -it may reach four or five times its original level-when they are cut or when their cardiac ends are paralysed by atropine; then 
the arterial pressure becomes so great as to expand passively the larger-sized arteries until they are seen to swell almost to bursting and to be proportionately elongated. The arterial contraction and the consequent rise of blood pressure are so decided that the strongest stimulation of the depressor nerve does not inhibit the contraction and reduce the pressure. Contraction of the spleen took place invariably and to a far greater degree than obtained with the limbs." Though we were unable to record any plethysmographic observations on the intestines, it may be fairly assumed that the great rise of the blood pressure which invariably follows the injection of the extract is in all cases due very largely to the contraction of the arterioles of the splanchnic area. We have proved that this contraction is not indirectly produced through the vasomotor centre, but is an immediate effect on the muscular tissue of the smaller arteries in mammals ; for it occurs equally well after destruction of the bulb or section of the cord, or, in the case of the arm, 'after severance of the brachial plexus, and in the frog with the brain and spinal cord completely destroyed.

The active principle is contained entirely in the medulla of the gland, is absent from the organ in advanced Addison's disease, is dialysable, and is not destroyed by boiling for a short time, nor by acids, nor by peptic digestion, but may be destroyed by long boiling or by alkalies; it is not neurine nor a salt of neurine, as was supposed by Cervello ${ }^{5}$ and MarinoZucco. ${ }^{6}$ It has so far eluded isolation. Mr. Moore has pointed out that it is probably identical with a substance which was first described by Vulpian ${ }^{7}$ as peculiar to the medulla of the suprarenals-its reactions not being afforded by any of the other glands-the solutions of which assume a rosered colour on exposure to the air or to oxidising reagents.

The intravenous iniection of digitalis and ergot produces quite insignificant efferts on the heart and the arteries by the side of those obtained from the smallest dose of the extract which we have employed. We have, however, found one substance which furnishes an effect practically identical, and that is nicotine. This fact is of some interest because Krukenberg in 1885 described a substance which he obtained from the suprarenal bodies having a chemical formula almost identical with that of pyridine. ${ }^{9}$ an 1 nicotine may be regarded as a pyridine derivative. The active principle of the adrenals is, lowever, not pyridine, for the observations of Lauder Brunton and Tunnicliffe ${ }^{10}$ showed that the physiological action of that body is quite different from that of the suprarenal extracts, but it is not improbable that it may eventually be shown to be an organic alkaloid the nucleus of which is pyridine.

Perlia os the ros ' intere sting and important fact in regard to the active pri ciple of the adrenals is the extreme minuteness of the dise that is necessary to produce the results just described, and in this respect it undoubtedly agrees with what is known in regard to the potency of nicotine. Our experiments have shown that so small a portion of the extract as is equivalent to $5 \frac{1}{2}$ milligrammes (o.0055 g.) of the dried gland will induce the maximum effect on the heart and the arteries of a dog weighing ro kilogrammes or $\frac{1}{2}$ milligramme for each kilogramme of body weight. How infinitesimally small, therefore, must be the proportion of the active principle injected in such a diminutive dose of the gland will be apparent when deductions are made for the cortex ( $\frac{3}{4}$ of the gland in weight) which we have proved to be devoid of physiological activity, and for the proteid and other material (io of the dried gland) which are not dialysable, so that it follows that a maximal effect is produced by not more than 14 millionths of a gramme of the active adrenal material per kilogramme of body weight. ${ }^{11}$

4 In a paper on the Rhythmic Contractility of the Spleen, read before t'ie Royal Society last February (BRITISH MEDICAL JOCRNAL. p. 396), Professor Schäfer and Mr. Moore showed that suprarenal extract caused an enormous contraction of the spleen followed by an increase of the rhythmic waves.

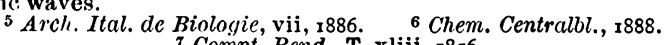$$
{ }_{7} \text { Compt. Rend., T. xliii. } 1856 \text {. }
$$

8 I refer particularly to the results of Mr. Moore's work on this subject carried out in the Physiological Laboratory of University Colloge, communicated to the Physiological Society, and published in the Journal of Plyysiology of last year.

$$
\begin{aligned}
& 9 \\
& \text { 10. Virchow's Archiv, p. ror, } 1885 \text {. }
\end{aligned}
$$

1! Sec Schiifer, British Nedical' JourNat, vol ii, isg:, p. $34 \mathrm{r}$.
The Effect of Adrenal Treatment on the Circulation.-It has been repeatedly observed that when circulatory compensation was impaired, as in anæmia and in other asthenic conditions, the exhibition of the adrenal preparation was followed in a few days by restoration of the postural radial measurements, and this observation has been repeatedly checked by the effects produced by omitting the treatment and by resuming it. The accumulation of a large number of concurring observations, in which small doses of the dried medulla of the gland ${ }^{12}$ have been prescribed in various forms of vasomotor (splanchnic) inadequacy has led to the conviction that suprarenal treatment in such cases is a useful addition to our therapeutic resources, and that it should be applied rather from this wider view, based on its physiological action, than merely from the exclusive one suggested by Addison's disease.

The foregoing observations tend to show that the ductless glands furnish to the blood certain materials of great potency which are necessary for the purpose of modifying in various ways the circulatory mechanism. It can, therefore, no longer be said that the whole of that office should be allotted to the nervous system. Though the glands yield internal secretions having an office subordinate to that of the nerves, they are nevertheless of great importance, is proved by pathological facts; and as their activity is not destroyed by digestion, an avenue is provided for the operation of their remedial power.

\section{Massage.}

The circulation of blood through the manipulated tissues is accelerated and increased. This fact has been clearly demonstrated experimentally on dogs and cats by Lauder Brunton and Tunnicliffe:

r. During the massage of muscles the flow of blood through them is increased.

2. Immediately after the cessation of massage an accumulation of blood occurs in the massaged muscles; this is rapldly followed by an increased flow through the muscles.

3. The massage of a considerable muscular area causes first a slight rise in the general blood pressure; this is followed by a fall which in ome cases amounts to one-fifth of the initial blood pressure.

The results of my observations in man coincide with these conclusions. The local effects on the radial pulse are easily learnt by applying the various forms of massage to the muscles of the forearm. The lighter and superficial manipulations-such as stroking, effeurage-and vibratory move ments or percussion-such as what is known as hacking, punctuation, slapping, etc.-raise the radial blood pressure and calibre; and the intermittent rolling and squeezing of the muscular tissue (pétrissage) reduce them.

The immediate effect of all the forms of massage is to raise the blood pressure and to enlarge the calibre of the artery. This preliminary stage is maintained for some time while tl.e more superficial and vibratory manipulations are being employed; but it is very transitory indeed when that furm c massage is applied which freely moves the muscular tissuf, and is quickly followed by a very decided and sharp reduction of the radial calibre.

From this it would seem probable that the first effect of massage in its various forms-and an effect which is, moreover, maintained throughout the application of the superficial and vibratory manipulations when limited in durationis to induce vasomotor contraction in the terminal vessels, and thus to raise the blood pressure and the calibre in the intermediate arterial tubing between them and the heart. This may, therefore, be regarded as the vasomotor tonic aspect of massage when that process assumes the forms of stroking, vibration, and percussion, which therefore raise arterial tension. When, however, the muscular tissue is freely manipulated and rolled the vessels therein dilate, and the flow of blood through them is increased and accelerated by the rapid manipulations acting in the manner of a supplementary heart, and the blood pressure and calibre of the

12 The medulla was rapidly dried at $38^{\circ} \mathrm{C}$., and immediately formed into pills with an impervious coating, so as to preserve the product from atmospheric changes.

${ }^{13}$ On the Effects of the Kneading of Muscles upon the Circulation, Local and General. By T. Lauder Brunton, F.R.S.: and F. W. Tunnicliffe. M.D., Journal of Physiology, vol. xvii, 1894. Kleen also showed that kneading of the muscles of a rabbit's leg produced a fall of the blood pressure (Skandinav. Archin $f$. Physiol.. 1, 247, 1887). These observations have rocently hron confirmed by Reid Hunt, Journal of Physiology, vol. Xviii, I $;$, p. $3^{3}$ 
radial and other systemic arteries fall. This is the yasomotor relaxant effect of massage which reduces arterial tension. When the muscles of all the limbs are manipulated the radial calibre and pressure fall. There may be perceived a larger ictus after the manipulations, but it is unsafe to infer therefrom that the radial calibre is increased; for arteriometric measurement has shown that when the pulse tension is reduced the pulse stroke generally feels larger, though the calibre of the vessel is actually diminished.

The diversion of the blood to the capacious periphery located in the muscles is no doubt a leading cause. But there is some evidence that points to the conclusion that this may not be the sole cause.

When vigorous massage is applied to the limbs and the back for twenty or thirty minutes, the normal postural variations of the radial calibre and pressure are very apt to become-if they do not invariably become-reversed, so that they measure less in the sitting than they do in the recumbent posture; and when this happens, it has been found that manipulation of the abdomen for eight or ten minutes restores the normal formula of the pulse.

It would, therefore, seem probable that massage, when confined to the systemic muscles, disposes to the accumulation of blood within the capacious venous system of the abdomen. Glovetzky made a large number of experiments on men and dogs to show the effects of abdominal massage on the circulation. He found that the upper half of the body increased in weight after the séance as shown by Mosso's balance, and plethysmographic measurements proved that during the sitting both the upper and lower extremities increased in volume, but returned to their normal size after the massage. The blood tension and the intracranial pressure invariably rose during the massage, and the elevation lasted for a certain period afterwards. ${ }^{14}$

But though a short application of abdominal massage may restore the normal contraction of the vessels supplied by the splanchnic nerves, its continuance beyond a very limited period is apt to defeat the aim in view; for then it is not improbable either that the contractility of the vessels becomes exhausted and dilatation of them is thus favoured, or the heart's action is lowered through reflex over-stimulation. So that it would seem to be best, as a rule, to apply massage to the abdomen in some of its vibratory forms, and for a brief period only, and after the general massage has been performed.

Massage and Exercise.-It has been pointed out that in exercise-local and general-the volume of the limb is considerably increased from the afflux of blood, and probably a!so from the infiltration of watery fluid from the gorged vessels into the substance of the muscles. Volumetric observation of the arm has shown that massage, when applied for a short time-for example, from 7 to ro minutes-generally induces a slight diminution of the limb. Therefore it would seem probable that any watery exudation that may flow from the blood is pressed forward from the lymph spaces into the lymphaties.

The practical outcome of these observations, which probably coincides in the main with the teaching of general experience in regard to massage, seems to be the following:

Massage should not be prescribed and performed in a mere perfunctory routine fashion, as is to be feared it, too often is but, like other therapeutic measures, it should be applied with due discrimination. The leading features of the circulation are the best indications for the selection of the kind of manipulations to be adopted for the limitation of the time of their application and for the distribution of them, whether mainly to the viscera or to the systemic muscles. When, for example, the vasotonic effects are sought for, the manipulations should nartake chiefly of the nature of vibration or percussion, and in those cases in which muscle kneading is resorted to, percussion should terminate the process. When, on the other hand, the aim is to reduce arterial tension and to relieve the ventricular effort, the deep movements should be preferred throughout, and should be applied only to the limbs and back, and the abdomen should not be manipulated.

Resistive Movements.

Certain exercises in which the operator ofters resistance are of special value in the development of the muscles and in the acquirement of muscular tone and vigour. The system of exercises of this character to which I refer is known as the Swedish system. Nearly fifty years ago, Saetherberg, of Stockholm, applied this method to the treatment of heart disease, and reported his results some fifteen years later $;^{15}$ and, following him, one or two other observers have shown the value of resistive gymnastics in cardiac disease. But the method has only of late years acquired prominence as a definite therapeutic mode of treating failing compensation of the heart since the brothers August and Theodor Schott modified it in certain respects, and applied it under proper restrictions, either in conjunction with or apart from the baths administered at Bad Nauheim.

The few observations to be adduced refer only to the physiology of these exercises, or rather as to how the results which are believed by some to be obtained from them are brought about. ${ }^{16}$ Two somewhat conflicting theories are entertained. On the one hand, Dr. Theodor Schott ascribes it to reflex stimulation of the cardiac inhibitory nerves, causing the heart to beat more forcibly and more slowly; on the other hand, Dr. Bezly Thorne, ${ }^{17}$ Sir William and Ir. John Broadbent ${ }^{2}$ refer it to dilatation of the arteries supplied to the muscles. I have merely to submit a few observations on several healthy subjects, ranging in age from 32 to 46 , which may be regarded as having a bearing on one of these views.

In one case, a healthy doctor, aged 37 . the five arm movenents were distributed over thirty minutes. the intervals of rest being equal to the duration of the exercises. At the termination of the first exercise the calibre was found to be two points and the pressure ten points higher; but after each of the four subsequent exercises the calibre and the pressure were observed to fall rapidly, and at the close of the observation the former was found to be nearly $x$ mm. and the latter fhirty points 82 (sitting).

In another case a healthy man, aged 46 . subjected for thirty minutes to the arm exercises, followed by forty minutes' rest, and then succeeded by the three trunk movements, there was also an initial rise of radial calibre and pressure, followed by a rapid fall: and after the long interval of rest this reduction was further inte
pulse rate fell from 84 to 74 (sitting).

pulse rate fell from 84 to 74 (sitting). In a subject, aged 26, the volume of the arm was measured before and after four arm exercises of two minutes each interrupted by one minute of rest. The initial volume 1,648 c.cm. Was increased to 1,696 e.em., for fifteen minutes, and was replaced by the resistive movements for ten minutes.

It has been observed that the increased volume caused by the resistive exercises may persist for several hnurs.' These observations in healthy subjects have shown that the slow resistive exercises produce the following effects on the limb and on the radial pulse:

r. An increase in the volume of the limb. 2. At

3. A reduction of the fiequency of the pulse.

The reduction of the radial pressure and calibre is no dout $t$ due to the diversion of a large volume of blood to the capecious muscular periphery. The immediate increase of $t$ ? $\theta$ volume of the limb is direct proof of this fact; but, as in ordinary exercise, the persistence of the enlargement fir many hours shows that it is not due entirely to the temporai filling of the vessels within the muscles, but probably arisi $s$ from an infiltration of fluid derived from the blood into tre muscular tissue. This system of regulative movements; therefore, produces all the local physiological effects of exer:cise; but it prominently differs from ordinary exercise in one respect-namely, in the slowing of the heart's action." In general exercise the condition of the radial artery does not truly indicate the degree of tension that exists within the central arterial system from the augmented propulsion of blood into it, for the blood pressure at the point of observation is reduced bo the dilatation of the branches which at hirher points convey the increascel flow of blood to the muscles. In the early stage of exercisi? the radial tension is increased; but this is transitory, and is replaced by a reduction when the muscles begin to monopolise the blood supply. Exactly the same thing occurs as a result

15 Hygiea, 1862; quoted by Dr. Hermann Nebel, of Frankfort. Lancet, vol. $\mathrm{i}, \mathbf{1} 896$.

16 It is beside my purpose to touch upon the disputed clinical questic as to whether either the immediate or the remote effect of the exercisf is to actually reduce the dilatation of the cardiac chamter's or only to apparently do $\_0$. 17 British Mrfical Journal. vol. i, i896, p. 653. 
of the resistive exercises. As a matter of fact, it is known that in general exercise there is an increase of the mean blood pressure, notwithstanding the enormous diversion of blood to the muscles. ${ }^{19}$ But if the resistive movement method is but a form of exercise, why should it slow the heart while exercise accelerates it? It may be presumed that there is in both a rise in the mean blood pressure. According to Marey's law, there should be in both a slowing of the heart's action. In exercise it is obvious that the operation of this law is disturbed by other concomitant conditions, which determine an acceleration of the vascular pump, and by the increase of the respiratory movements. Now, when the resistive exercises are practised, the greatest care is taken not to allow the movements to. accelerate the patient's breathing, and he is directed to breathe calmly and regularly; so there is eliminated from this mode of exercise the main, if not the sole, cause of the acceleration of the heart which is invariably present in ordinary exercise; and the slowing of the vascular pump is just as likely, perhaps more likely, to be due to a slight rise in the aortic pressure as to reflex cardiac inhibition (the theory of Dr. Schott). The former view, which was pointed out by Dr. Saundloy, ${ }^{20}$ is more in accord with our knowledge of the physiology of exercise than the latter; and, moreover, it affords a reason why the exercises are regarded by Dr. Theodor Schott and others as unsuited to cases of myoearditis, of aortic aneurysm, and of advanced arterio-sclerosis.

The examination of the radial pulse by means of the finger and of the sphygmograph affords but equivocal evidence bearing on this point; and from what we know of the anatomical relations of the radial artery to the muscles, and of the pliysiological influence of the circulation through the muscles on the radial tension, it becomes, I think, somewhat doubtful if the radial pulse can afford trustworthy evidence of any alteration in the blood pressure which may be induced in the large arteries by the exercises.

It should be observed that the retardation of the heart's action, as well as the copious delivery of blood into the muscular periphery, must greatly limit and reduce any rise of arterial blood pressure that may take place. Hence these modulated movements throw less strain on the central arteries than ordinary exercise. 'The slowing of the heart is, therefore, a favourable sign; for it is an indication that the rise of blood pressure is being reduced by inhibition, and that the ventricle is not being subjected to an injurious strain by the exercises, and is duly responding to them in a more complete delivery of the blood.

Doubtless the immediate relief to the loaded heart afforded by this therapeutic method should be ascribed to the aspiration of a large volume of blood into the capacious muscular periphery by means of the tonic contraction of all the systemic muscles, so gentle and so maintained as just to fall sliort of stimulating the respiratory centre. ${ }^{21}$ The blood thus determined to the periphery must gradually return to the heart; and if the relief obtained is entirely due to this diversion of the blood, it will be of comparatively short duration. But there is some reason to believe that the volume of the blood is reduced during its sojourn through the muscles, and if the fluid withdrawn from the blood lodges for some time in the muscular tissue, the intervals of relief should be prolonged.

The total outcome, therefore, of the exercises is a transference of blood from the venous to the arterial side of the circulation, and a probable reduction of the volume of the blood.

I think it is not improbable that under physiological conditions the ventricular output is not, as a rule, ample enough to maintain the normal volume of the blood in the arteries while they are being largely depleted by the increased drain into the muscles in a state of vigorous contraction; whereas, when the ventricle is dilated, which involves dilatation of the other chambers of the heart, and can be incited to throw off the augmented load of residual blood, the arterial tension may rise in spite of the increased flow into the muscles.

19 A Textbook of Physiology, by Professor M. Foster, F.R.S., p. 350 20 BRITISH MEDICAL JOURNAL, 1895 , vol. ii, p. ro8

21 Dr. Harry Campbell has also pointed out that the total delivery of blood to the right heart is lowered in consequence of the contraction of the muscles compressing the veins (splanchnic and systemic) (BRITISH MEDICAL JOURNAL, vol. ii, r895, p. 1325).
This strictly physiological mode of developing and invigorating the walls of the heart, when applied with proper care and discrimination, is a therapeutic method of some value, and is a distinct addition to our resources for the treatment of failing compensation of the heart. I have observed several times that it has restored compensation after the complete failure of drugs, and that it is in suitable cases a useful auxiliary to other treatment. Like any other remedial method of decided power, it may do harm when inappropriately or improperly applied. In all cases I have found that the greatest caution should be exercised-especially in the early application of the method-to reduce the rate of the exercises to a minimum. There is often a disposition on the part of the operator to quicken the speed; that should always be deprecated unless this acceleration is fully warranted by the improvement attained.

There is no reason why the principle which underlies this physiological method of treatment-namely, tonic contraction of successive groups of muscles-should not be attained in other.ways and especially by mechanical means. Much may be done by the patient himself without an operator; and it has been found that auto-resistive exercises are useful in maintaining the advantage gained in the first instance, by the ordinary application of the method.

Baths.

The influence of baths on the circulation is too large a subject to be dealt with now. Apart from the modifying influence exerted by the constituents of mineral waters, it may be said that the leading effects of baths on the circulation are due to temperature; and as the results of observation with the arteriometer coincide with the generally accepted views on the influence of baths and temperature on the vasomotor system, I will merely state the conclusions which they support.

Hot immersion baths (from $100^{\circ}$ to $105^{\circ} \mathrm{F}$.) quickly reduce the radial calibre and pressure from the rapid dilatation which takes place in the arterioles and the cutaneous periphery. From this cause the volume of a limb taken immediately after the immersion is found to be considerably increased, but the increment rapidly subsides. In one example, the initial volume of $x, 640$ c.cm. was raised by the hot bath to 1,744 c.ell:, The enlargement is obviously due to a temporary turgescence of vessels, The enlargement is obviously due to a temporary

and therefore differs from that caused by exercise. Warm inmersion baths (from $96^{\circ}$ to 10 $^{\circ} \mathrm{F}$ ) enlarge the radial calibre, though after a certain time the peripheral ve
filled, and the radial is consequently reduced.

filled, and the radial is consequently reduced. Turkish baths, like hot immersion baths, quickly congest the cutaneous peripheral vessels and the veins, and consequently reduce the arterial pressure. When followed by the cold shower the arterial pressure immeguite attain its former size. It will be shown in the fourth lecture that quite attain its former size. It will be shown in the

Hot Footbath.-The well-known revulsive action of the hot footbath in reducing the arterial fulness in the upper part of the body has been illustrated by the arteriometer, for it has shown that an immersion of twenty minutes may reduce the radial diameter from $2.2 \mathrm{~mm}$. to $\mathrm{x} .3 \mathrm{~mm}$ The same fact was demonstrated by Winternitz as the result of the war'm sitz bath, for he found that the plethysmograph indicated a reduction o thesesze of the arm ; 22 and Schuller observed that the vessels of the exposed pia mater of the rabbit were diminished in

he extent of the body immersed in warm water.23 Cold Baths. - It has been observed that immersion in plain water of temperature below $9^{\circ} \mathrm{F}$. produces contraction of the systemic arteries within three minutes, the amount of contraction being proportionate to the reduction of the temperature. It would seem from the experiments of schüller that the blood thus diverted from the outer vascular area congests the vessels within; for on immersing rabbits into cold baths he observed dilatation of the vessels of the pia mater (exposed by trephining) in proportion to the extent of immersion. This diversion of the blood from the surface is. however, but temporary, and is followed by increased fulness of the systemic vessels after the immersion is over, or even while it continues if the subject is very vigorous. The local application of cold water-as in the cold sitz bath-is also of considerable therapeutic power when the principal aim is to divert an increased quantity of blood to the brain and the cord, as when the vasomotor mechanism is impaired and gravitation determines anæmia of the nerve centres. According to the observations of Winternitz, the cold sitz bath increases the volume of the arm as registered by the plethysmograph; so that it possesses the power of in creasing the distribution of blood in the upper parts of the body. We have here a reasonable explanation of the clarifying effect of the cold anæmia from impaired vasomotor compensation.

22 Ueber thermische Wirkungen auf die Blutvertheilung in die Hydrotherapie, B. i, 1890 .

23 Deutsche Archiv für klinische Medicin, B. xiv. A useful epitome of the experimental work on thermal therapeutics by Naumann, Schüller, and De Mosso in animals; and by Winternitz, Istamanoff, Mosso, and Ber esis in mon will be found in Professor Hayem's Lecons de Therapeutique. gesis in man will be found in Professor Hayem's Lecons de Therapeutique by Dr. Hobart Amory Hare." Edinburgh : Pentland. 
Shower, Needle, and Douche Baths.-The influence of temperature per se is greatly modified by the mechanical agency of percussion and vibration, so that when the water is divided into innumerable fine jets which impinge all over the surface of the body with a certain degree of force, or when it is applied in the form of the travelling douche accompanied by a vibratory form of massage, the vasomotor system is much more powerfully affected than by any form of still bathing, even though the water be charged with carbonic acid or saline matter. The therapeutic advantages of this form of bathing are very considerable, for it can be made to furnish all the tonic effects of cold bathing along with an agreeable temperature that may be adapted to individual requirements. A mire intelligent appreciation of the curative value of this and of similar forms of tonic bathing now prevails at many health resorts, where in times gone by a course of bathing often simply meant soaking in hot water with the consequent impairment of the vasomotor mechanism. The massage, douche, and needle bath is the form of bathing best of all adapted to counteract the enervating effects of city life with all its attendant worries and anxieties; and were it established in our large centres it would, I am persuaded, prove to be a boon to many sufferers from nervous exliaustion, and would become even more popular and valuable than the Turkish bath.

Carbonic acid and saline baths also belong to the stimulant form of bathing.

\section{CaUsation OF THE DOUBLE FIRST CaRdiaC SOUND.}

By E. M.BROCKBANK, M.D.Vict., M.R.C.P.LoND., Late Resident Medical Officer, Royal Infirmary, Manchester.

By a double first sound I mean that physical sign which is also known as a reduplicated first sound, and which occurs most typically and most commonly in cases of high arterial tension. This double first sound is sometimes wrongly referred to as a bruit de galop, for in the former, the component sounds come closely together, and can be distinctly and undoubtedly placed in their true position, namely, ventricular systole, in the cardiac cycle, whereas there is room for much divergence of opinion concerning the timing of the sounds which form the true bruit de galop.

My remarks in this paper will be confined to the double first sound alone, and I shall not at present touch upon the bruit de galnp at all, although I consider that its explanation is allied to that which I shall suggest to be the cause of the double first sound.

\section{Theories as to its Causation.}

My attention has been directed to the first sound more with a view to the elucidation of its physical causation thin to the pathological conditions of the body generally in which it occurs. This latter part of the subject has been dealt with recently by Kauffmann. ${ }^{1}$ As a result of observations on 34 cases, Kauffmann found that the chief ailments in which a double first sound may occur are chronic Bright's disease; cardiac dilatation, especially the left ventricle; weakness from dyspepsia or from a previous acute illness; displacement of the heart from chronic causes; and occasionally bronchitis and emphysema. I have met with the abnormal sign in Bright's disease (acute and chronic), anæmia, chronic rheumatism with anæmia, and debility from various causes.

As a result of my own experience, I am of opinion that the phenomenon may occur in any case in which the cardiac muscle is actually weak (anæmia, cardiac dilatation, etc.), or only weak relatively to increased arterial tension (Bright's disease). Several theories have been from time to time advanced in explanation of the double first sound of the heart. The most important are referred to fully by Sansom, ${ }^{2}$ but not one of these is to my mind satisfactory. They are as follows :

r. Asynchronous , closure of the mitral and tricuspid valves, and from 1.:Asynchronous : closure of the mitral and tricuspid
asynchronous action of the ventricles (Potain, Barr).

(he mitral and tricurspid valves (Guttmann).

3. Resolution of the first sound into its elements of muscle contraction and valve tension (Hayden). 4. The rapidly following sounds first of auricular then of ventricular
systole (Geo. Johnson).

5. A sliock caused at the presystolic period of the ventricular action by the auricular, systole which suddenly, by contrecoup from the apex of by the auricular, systole which suddenly, by contrecoup from the apex of
the ventricle, makes tense the auricular and ventricular valves (Sansom).

There, is very little evidence in support of any one of these theories, and it is in consequence difficult to criticise them. I shall not attempt to do so, but will simply bring forward what I consider to be a more probable cause of the double

\footnotetext{
2 Diagncsis of Disease of the Heart and Thoracic Aorta, 1892, p. 209 , et seq.
}

first sound, and mention physiological and clinical facts in its support.

\section{A Suggested Explanation.}

The double first cardiac sound is caused by failure on the part of the musculi papillares to retain under the intra. ventricular blood pressure the contracted and shortened condition which they assume during the ventricular systole of the cardiac cycle. This failure on the part of the papillary muscles allows the curtains of the auriculo-ventricular valves to rebound, and thereby cause a sound of sudden and forcible tension of the valve curtains and the chordæ tendineæ. The abnormal sound forms the second portion of the double first sound, the first portion being due to the causes which produce the normal first cardiac sound. The failure of the musculi papillares may take place in either or both of the ventricles and affect one or both valves.

Physiological Evidence.

I shall, in the first place, refer to the part played by the musculi papillares in aiding the expulsion of blood from the ventricles during their systole. Roy and Adami, ${ }^{3}$ by means of an ingenious apparatus, took simultaneou $*$ tracings of the action of the ventricle wall and of the musculi papillares during the cardiac cycle of a dog. As a result of these observations they divide the ventricular systo'e into five, and the diastole into three, phases.

Phases of Ventricular Systole.

Ventricle wall contracts, but the musculi papillares are at rest.

2. Musculi papillares carry out the first rapid part of their contractioa: ventricle wall at rest.

3. Shortening of the musculi papillares still continues, but less rapidly 3. Shortening of the musculi papillares still continues, but less rapidy
han in Pliase 2 ; ventricle wall contracts further, but not so rapidly as in than in

4. Musculi papillares and ventricle wall remain contracted, but do not undergo further shortening.

5. Musculi papillares expand rapidly whilst the ventricle wall remains contracted.

PHases of Ventriculat D
r. Rapid and uniform expansion of ventricle

r. Rapid and uniform expansion of ventricle. 2. Slowing or arrest of expansion if the beod supplying the ventricle by the elastic quansion of Phase

the elastic expansion of Phase 1 . from contraction of veins and auricles reaches the ventricle and causes or allows the final expansion of the reaches the ventricle, and causes or allow
ventricular wall which precedes systole.

These eight phases then show the parts played by the cardiac muscle during ventricular systole and diastole.

The variation which the intraventricular pressure undergoes during the cardiac cycle wos also investigated by Roy and Adami. The conclusions th y come to may be stated shortly. "The intraventricular pressure curve is made up of tw. curves.......which do not bear a constant relation to each other."

The first and larger wave is due to the contraction of the ventricles in Phase 1 . At first the rise of pressure in this wave is slow owing to the passive stretching of the musculi papillares, but its completion is rapid. Upon this wave is superposed another of shorter duration $(2-3)$, which indicates the maximum intraventricular pressure. This second short wave is due to the contraction of the musculi papillares. The intraventricular pressure then falls rapidly for a time, owing to the escape of blood into the anrta; but by the final combined contraction of the ventricle and musculi papillares it is maintained at a high level until all the blood is expelled from the ventricle into the aorta:

Positions Assumed by the Auriculo-Ventricular Valves during the Cardiac Cycle.

During Phase I of ventricular systole the curtains of the auriculo-ventricular valves are rendered tense and are bulged upwards towards the auricle. "The edges of the valves may even be pushed away from the point of origin of the papillary. muscles by the rise of intraventricular pressure. This must be due to passive stretching of the chordæ tendineæ and the musculi : papillares." $*$ During Phase 2 the valve curtains are pulled down towards the apex of the ventricle by the musculi papillares diminishing the amount of the bulge into the auricular navity. During Phases 3 and 4 the relative position of the valve curtains to the apex of the heart remains much the same as in Phase 2, and during the papillary relaxation in Phase 5 , the curtains retire towards the auricle. During 